09

\title{
Анизотропия оптических, электрических и фотоэлектрических свойств модифицированных фемтосекундным лазерным облучением пленок аморфного гидрогенизированного кремния
}

\author{
(C) Д.В. Амасев, ${ }^{1}$ М.В. Хенкин, ${ }^{2}$ R. Drevinskas, ${ }^{3}$ P. Kazansky, ${ }^{3}$ А.Г. Казанский ${ }^{2}$ \\ ${ }^{1}$ Институт общей физики РАН, \\ 119991 Москва, Россия \\ ${ }^{2}$ Московский государственный университет им. М.В. Ломоносова, \\ 119991 Москва, Россия \\ ${ }^{3}$ Optoelectronics Research Centre, University of Southampton, \\ Southampton SO17 1BJ, UK \\ e-mail: kazanski@phys.msu.ru
}

(Поступило в Редакцию 22 июня 2016 г.)

Два типа независимых анизотропных структур были сформированы одновременно в результате структурной модификации пленок аморфного гидрогенизированного кремния облучением фемтосекундного лазера: структура с периодом в единицы-десятки микрометров и структура с периодом в сотни нанометров. Различие механизмов возникновения данных структур позволяло желаемым образом формировать их относительную ориентацию. Обе структуры независимо влияли на оптические свойства модифицированных пленок, приводя к дифракции проходящего света и возникновению поляризационной оптической чувствительности пленок. Проводимость модифицированных пленок коррелировала с взаимной ориентацией анизотропных структур, в то время как взаимосвязи фотопроводимости с оптическими особенностями модифицированных пленок обнаружено не было.

DOI: 10.21883/JTF.2017.06.44516.1950

\section{Введение}

Пленки нанокристаллического гидрогенизированного кремния $(n c-\mathrm{Si}: \mathrm{H})$ используются для создания на их основе тонкопленочных солнечных элементов, полевых транзисторов [1], а также тонких оптических элементов, называемых метаповерхностями [2,3]. Одной из широко применяемых методик получения пленок $n c-\mathrm{Si}: \mathrm{H}$, позволяющей формировать их на „низкотемпературных“ подложках, является метод лазерной кристаллизации пленок аморфного гидрогенизированного кремния $(a-\mathrm{Si}: \mathrm{H})$ [4]. Использование фемтосекундного лазерного излучения с энергией кванта в области края поглощения $a-\mathrm{Si}: \mathrm{H}$ позволяет провести однородную по толщине кристаллизацию пленки аморфного кремния без заметного разогрева подложки, на которую нанесена данная пленка. Одним из преимуществ лазерной кристаллизации пленок $a$-Si:H является то, что помимо модификации структуры пленок, происходит наноструктурирование их поверхности [5]. В результате этого существенно уменьшается отражение падающего света, что должно привести к увеличению эффективности его преобразования в солнечных элементах.

Для получения пленки $n c-\mathrm{Si}: \mathrm{H}$ необходимой площади при лазерной кристаллизации проводят сканирование поверхности $a-\mathrm{Si}: \mathrm{H}$ лазерным пучком. При этом на поверхности пленки формируется „микрометровая“ периодическая структура с периодом, соизмеримым с шириной лазерного пучка и/или шага сканирования, составляющих, как правило, единицы или десятки мик- рометров. Помимо этого, проведенные в работе [3] исследования показали, что при фемтосекундной лазерной кристаллизации $a-\mathrm{Si}: \mathrm{H}$ на поверхности пленки возможно формирование „нанометровой“ периодической структуры, не связанной со сканированием поверхности пленки $a-\mathrm{Si}: \mathrm{H}$ лазерным пучком. Авторы [3] показали, что структура поверхности модифицированной фемтосекундным лазерным излучением пленки $a$-Si:H существенно зависит от использованной интенсивности лазерного пучка. При этом в некотором интервале интенсивностей лазерных импульсов формируется периодическая структура с периодом 200-300 nm в направлении, совпадающим с направлением поляризации использованного лазерного излучения. Данная структура состоит из кластеров размерами в сотни нанометров, вытянутых перпендикулярно направлению поляризации использованного лазерного излучения. Формирование указанных планарных анизотропных структур приводит к появлению двулучепреломления в пленке и возникновению эффекта поляризации света в видимой области спектра [3].

Как отмечено выше, лазерная кристаллизация пленок $a-\mathrm{Si}: \mathrm{H}$ используется для создания различных электрических и фотоэлектрических структур. Формирование в определенных условиях при фемтосекундной лазерной кристаллизации $a$-Si :H рассмотренных выше двух типов периодических структур микрометрового и нанометрового размеров должно приводить к анизотропии оптических, электрических и фотоэлектрических свойств таких пленок. Поэтому представляет интерес исследование 
данных свойств в модифицированных лазерным излучением пленках $a$-Si:H, демонстрирующих рассмотренную выше периодическую структуру. Результаты данных исследований представлены в настоящей работе.

\section{Исследованные образцы и методика измерений}

Нелегированные пленки $a-\mathrm{Si}: \mathrm{H}$ толщиной $300 \mathrm{~nm}$ наносились на подложку из кварцевого стекла методом плазмохимического осаждения из газовой фазы, состоящей из объемной смеси 25\% моносилана и $75 \%$ аргона при температуре подложки $250^{\circ} \mathrm{C}$. Для облучения пленок фемтосекундными лазерными импульсами использовался комплекс на основе $\mathrm{Yb}: \mathrm{KGW}$-лазера (длина волны излучения $1030 \mathrm{~nm}$, длительность импульсов $360 \mathrm{fs}$, частота следования импульсов $100 \mathrm{kHz}$ ). Энергия лазерных импульсов соответствовала условиям формирования структуры, демонстрирующей оптическую анизотропию, и составляла $0.125 \mu \mathrm{J}$. Модификация структуры пленки $a-\mathrm{Si}: \mathrm{H}$ проводилась в результате сканирования поверхности пленки $a-\mathrm{Si}: \mathrm{H}$ со скоростью $0.2 \mathrm{~mm} / \mathrm{s}$ „гребенкой“, состоящей из 10 лазерных пучков, расположенных на расстоянии $20 \mu \mathrm{m}$ друг от друга. Каждый из пучков при заданной энергии лазерных импульсов модифицировал полосу пленки шириной $8 \mu \mathrm{m}$. Шаг сканирования гребенкой составлял $5 \mu \mathrm{m}$.

Структура поверхности модифицированных пленок анализировалась с помощью сканирующего электронного микроскопа (Zeiss Evo 50) и микрорамановского спектрометра (Horiba Jobin Yvon HR800) с использованием излучения с длиной волны $488 \mathrm{~nm}$. Для проведения электрических и фотоэлектрических измерений на поверхности модифицированных кремниевых пленок напылялись алюминиевые контакты длиной $4 \mathrm{~mm}$ и расстоянием между ними $0.5 \mathrm{~mm}$. Измерения проводимости и фотопроводимости проводились в вакууме при остаточном давлении $10^{-3} \mathrm{~Pa}$ после отжига пленок при $170^{\circ} \mathrm{C}$ в течение $5 \mathrm{~min}$.

\section{Результаты и их обсуждение}

На рис. 1 показаны полученные с помощью сканирующего электронного микроскопа картинки поверхности пленки, модифицированной фемтосекундным лазерным излучением. Как видно из рис. 1, $a$ на поверхности пленки можно наблюдать сформированные полосы в направлении сканирования лучей лазера. В то же время из рис. $1, b$ и $c$ видно, что при использованной энергии лазерного импульса на поверхности пленки формируется также структура, состоящая из кластеров размерами в сотни нанометров, вытянутых преимущественно в направлении, перпендикулярном направлению поляризации, использованного лазерного излучения. На рис. 1
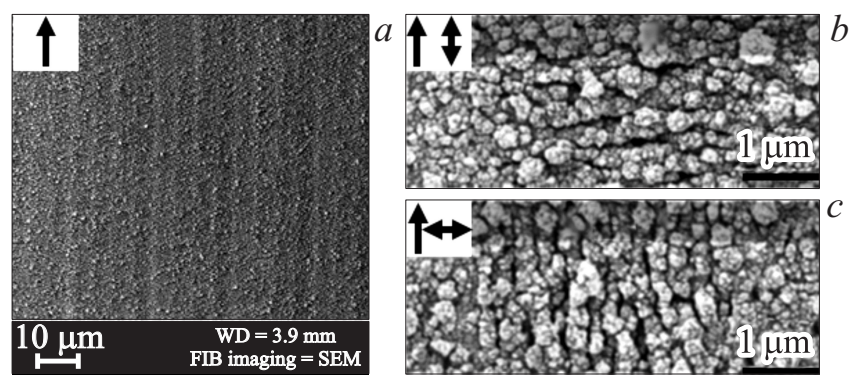

Рис. 1. Фотографии поверхности модифицированных пленок, полученные с помощью сканирующего электронного микроскопа с малым $(a)$ и большим $(b, c)$ разрешеением.
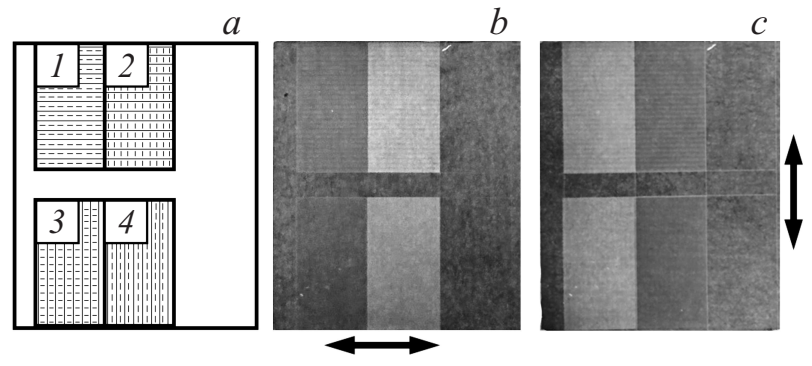

Рис. 2. Схема расположения исследованных образцов на подложке $(a)$ и фотографии пропускания через них поляризованного белого света $(b, c)$. Направления поляризации падающего на образцы света показаны двойной стрелкой.

стрелками и „Двойными“ стрелками показано соответственно направление сканирования лазерным лучом и направление его поляризации.

Таким образом, использованная нами методика модификации структуры пленок $a-\mathrm{Si}: \mathrm{H}$ с помощью фемтосекундного лазерного излучения приводит к формированию структурной анизотропии микронного размера в результате сканирования луча лазера и анизотропии нанометрового размера, определяемой поляризацией лазерного луча, модифицирующего структуру пленки.

Поскольку причины формирования нанометровой и микрометровой периодических структур различны, их взаимная ориентация может быть задана произвольным образом. Нами были исследованы 4 образца с различным взаимным расположением периодических структур и направлением протекания тока. Схематически структура исследованных образцов изображена на рис. 2, $a$, где направление микрометровых структур обозначено сплошными линиями, а направление нанометровых структур - штриховыми линиями. Ток во всех образцах, показанных на рис. 2, a, протекает в горизонтальном направлении.

Нанометровая и микрометровая периодические структуры пленок приводили к анизотропии оптических свойств исследованных образцов. На рис. $2, b$ и $c$ показаны фотографии пропускания белого поляризованного 


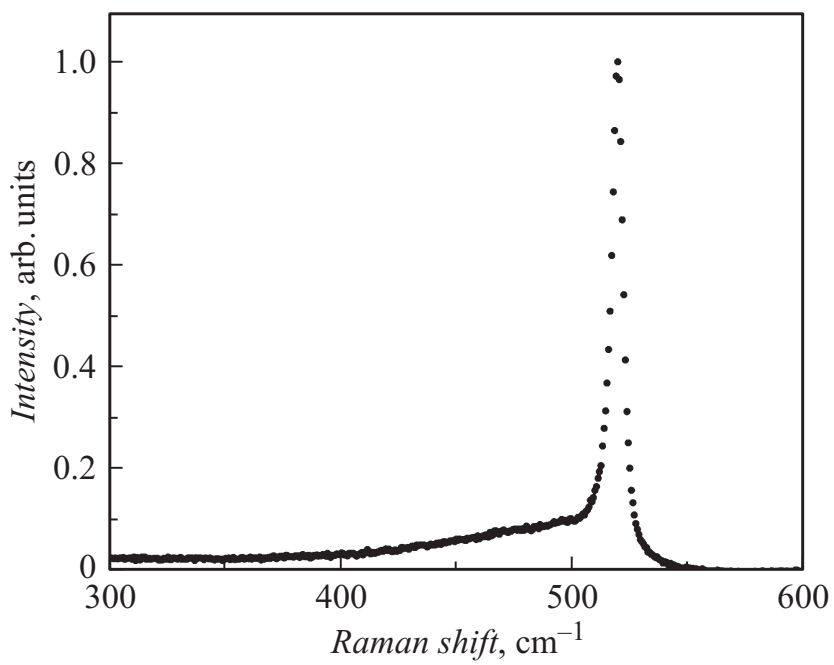

Рис. 3. Спектр комбинационного рассеяния в исследованных образцах.

света (направление поляризации показано на рисунке двойной стрелкой) через области пленки, обработанные при различных направлениях сканирования лазерного пучка и при различных направлениях вытянутых нанометровых кластеров. На этом же рисунке видна область пленки $a-\mathrm{Si}: \mathrm{H}$, необработанной лазерным излучением. Как видно из рис. 2, формирование анизотропной структуры нанометрового масштаба, определяемой поляризацией лазерного луча, приводит к возникновению „поляризационного“ эффекта в пленке. Пропускание излучения, поляризованного вдоль протяженных кластерных образований, меньше, чем пропускание излучения, поляризованного поперек протяженных кластерных образований. Заметим, что близкие нашим результаты были получены в работе [6] для пленок пористого кремния, в котором была сформирована анизотропная структура, c „вытянутыми“ в одном направлении нанокристаллами. Полученные результаты авторы [6] объясняли меньшей величиной электрического поля внутри нанокристаллов в случае поляризации электромагнитной волны перпендикулярно вытянутому направлению нанокристаллов. Таким образом, анизотропия структуры нанометрового размера, показанная на рис. $1, b$ и $c$ приводит к возникновению поляризационного эффекта в пленке.

В то же время периодическая структура микронного масштаба, возникающая в результате процесса сканирования, проявлялась в эффекте дифракции видимого света, наблюдаемой в направлении, перпендикулярном направлению сканирования. Причем структура дифракционных максимумов, наблюдаемых на дифракционных картинах наших пленок, может быть интерпретирована как наложение рефлексов от трех дифракционных решеток с периодами 5, 20 и $200 \mu \mathrm{m}$. Данные периоды возникают в результате использованной нами процедуры сканирования пленок: 1) $5 \mu \mathrm{m}-$ смещение между двумя полосами сканирования; 2) $20 \mu \mathrm{m}$ - расстояние между соседними лучами гребенки; 3) $200 \mu \mathrm{m}$ - период трансляции всей гребенки. Полученный результат свидетельствует о том, что, несмотря на перекрытие между соседними полосами сканирования, структура пленок получается „оптически неоднороднойс“.

Фазовый состав кремниевых пленок анализировался при помощи спектроскопии комбинационного рассеяния света (КРС). Спектры КРС, измеренные со стороны поверхности модифицированной пленки и со стороны подложки, были близки друг другу, что свидетельствует об однородности структуры модифицированной пленки по ее толщине. В качестве примера на рис. 3 представлен спектр комбинационного (рамановского) рассеяния, измеренный со стороны поверхности пленки. Как видно из рисунка, в рамановском спектре модифицированной пленки наблюдается интенсивная линия при $520 \mathrm{~cm}^{-1}$, соответствующая нанокристаллической фазе структуры, и широкая полоса, в которую дает вклад сигнал с максимумом при $480 \mathrm{~cm}^{-1}$, соответствующим аморфной фазе структуры [7]. Анализ полученных спектров рамановского рассеяния с использованием методики, описанной в работе [7], позволил оценить объемную долю нанокристаллической фазы в пленке, которая составила $72 \%$. Заметим, что данная величина представляет собой усредненное значение по площади, соответствующей диаметру луча лазера рамановского спектрометра, составляющего $1 \mu \mathrm{m}$.

Проведенные измерения показали, что планарная структурная анизотропия полученных образцов приводит к анизотропии их проводимости $\left(\sigma_{d}\right)$. На рис. 4 показаны температурные зависимости проводимости, полученные для образцов, у которых отличалась ориентация анизотропных микрометровой и нанометровой структур относительно направления протекания тока. Для образцов 1 и 2 направление тока совпадало с

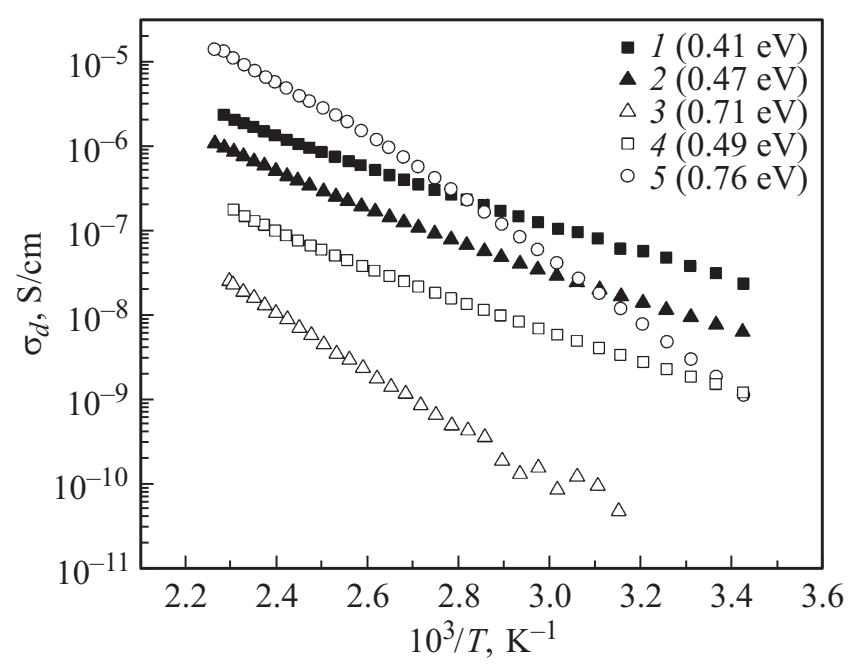

Рис. 4. Температурные зависимости проводимости исследованных образцов (1-4) и необработанной пленки $a$-Si:H (5). Номера кривых (1-4) соответствуют номерам исследованных образцов. 


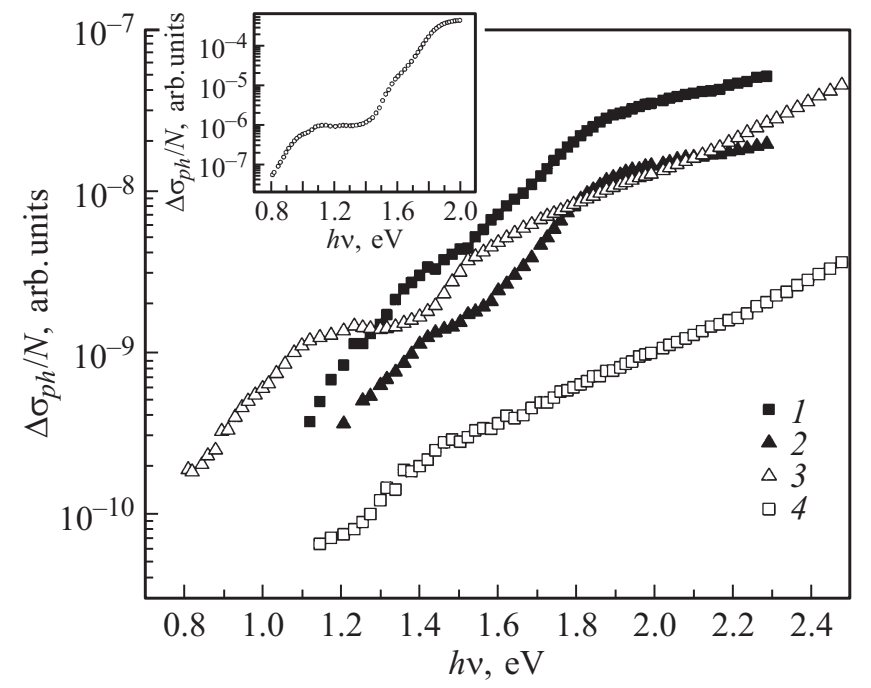

Рис. 5. Спектральные зависимости фотопроводимости исследованных образцов. Номера кривых (1-4) соответствуют номерам исследованных образцов. На вставке показана спектральная зависимость фотопроводимости необработанной пленки $a-\mathrm{Si}: \mathrm{H}$.

направлением полос сканирования, в то время как для образцов 3 и 4 направление тока было перпендикулярно направлению полос сканирования. На рис. 5 также для сравнения показана температурная зависимость проводимости необработанной лазерным излучением области пленки $a-\mathrm{Si}: \mathrm{H}$. Как видно из рисунка, для всех исследованных образцов температурные зависимости проводимости вблизи комнатной температуры близки к активационным. Значения энергии активации $E_{a}$ представлены на рисунке. Для пленки аморфного кремния до ее обработки излучением фемтосекундного лазера энергия активации $E_{a}$ составляет $0.76 \mathrm{eV}$. После модификации структуры $a-\mathrm{Si}: \mathrm{H}$ излучением лазера энергия активации уменьшается и составляет $0.4-0.5 \mathrm{eV}$. Данное значение характерно для пленок нелегированного нанокристаллического кремния. Значения проводимости модифицированных пленок зависят от ориентации анизотропной структуры микрометрового и нанометрового размеров относительно направления протекания тока. Максимальная проводимость наблюдается для образца 1, у которого направление полос, связанных со сканированием лазерного луча, и направление вытянутых нанометровых кластеров, определяемых поляризацией луча фемтосекундного лазера, совпадают с направлением протекания тока. В то же время минимальная проводимость наблюдается для образца 4, у которого направление полос, связанных со сканированием, и направление вытянутых нанометровых кластеров, определяемых поляризацией луча фемтосекундного лазера, перпендикулярны направлению протекания тока. Уменьшение проводимости при ориентации протяженных кластеров и/или полос сканирования перпендикулярно протеканию тока, повидимому, связано с увеличением вклада в измеряемую проводимость участков с малой проводимостью. Эти участки могут быть связаны как с промежутками между нанокристаллическими кластерами в модифицированных пленках, так и с участками пленки с большой долей в их структуре аморфного кремния, наличие которого подтверждают спектры рамановского рассеяния. Данные участки могут находиться на границах полос сканирования.

На рис. 5. показаны спектры фотопроводимости $\Delta \sigma_{p h}=\sigma_{p h}-\sigma_{d}$, (где $\sigma_{p h}-$ проводимость образцов при их освещении), полученные для модифицированных пленок и нормированные на число падающих квантов $(N)$. Для образцов 1 и 2 направление фототока совпадало с направлением полос сканирования, в то время как для образцов 3 и 4 направление фототока было перпендикулярно направлению полос сканирования. Для сравнения на этом же рисунке на вставке показана спектральная зависимость фотопроводимости пленки $a$-Si: H до ее облучения излучением лазера. Существенное уменьшение фотопроводимости пленки $a-\mathrm{Si}: \mathrm{H}$ после ее лазерной обработки, по-видимому, связано с дегидрогенизацией пленки в результате ее лазерной кристаллизации, что было отмечено в работе [8]. Эффузия водорода должна приводить к увеличению концентрации оборванных связей, являющихся основными центрами рекомбинации неравновесных носителей заряда в аморфной и нанокристаллической фазах модифицированных пленок. Из рисунка также видно, что величина фотопроводимости модифицированных лазерным излучением пленок зависит от ориентации микрометровой и нанометровой структур относительно направления протекания тока. В то же время мы не наблюдали корреляции в изменении фотопроводимости и проводимости для пленок с различной структурой (cp. рис. 4 и 5). Это может быть связано с тем, что в отличие от темновой проводимости, которая в основном определяется процессом переноса носителей заряда, фотопроводимость определяется также процессом генерации и рекомбинации неравновесных носителей заряда в исследованных образцах, состоящих из кластеров и имеющих к тому же двуфазную аморфно-нанокристаллическую структуру.

Сложная „Планарная“ и сложная фазовая структуры исследованных образцов приводят к сложной форме полученных спектральных зависимостей фотопроводимости, которые, по-видимому, являются комбинацией спектральных зависимостей фотопроводимости аморфной и нанокристаллической фаз, формирующих образцы. Как отмечено выше, анизотропия наноразмерной кластерной структуры исследованных образцов приводила к возникновению у них оптических поляризационных свойств. В то же время проведенные исследования не обнаружили влияния поляризации падающего на образцы света на величину фотопроводимости и ее спектральную зависимость. Можно предположить, что это связано с тем, что оптическая анизотропия вызвана взаимодействием света с состоящими из нанокристаллов 
кремния кластерами анизотропной формы. В то же время вследствие большой концентрации рекомбинационных центров на границах нанокристаллов они не дают вклада в фотопроводимость, которая в основном определяется аморфной фазой структуры. На это указывает наблюдаемое увеличение фотопроводимости вплоть до энергий, соответствующих ширине щели подвижности $a-\mathrm{Si}: \mathrm{H}(1.8 \mathrm{eV})$. Заметим, что определяющая роль аморфной фазы в фотопроводимости модифицированных лазерным излучением пленок $a-\mathrm{Si}: \mathrm{H}$ была отмечена в работе [9].

\section{Заключение}

Таким образом, проведенные исследования показали, что фемтосекундная лазерная обработка пленок аморфного кремния приводит к появлению двух типов периодических поверхностных структур, геометрия которых может контролироваться независимо друг от друга. Периодические структуры микрометрового масштаба, связанные с трансляцией лазерных лучей, приводят к дифракции видимого света. Периодические структуры нанометрового масштаба, направление которых определяется направлением поляризации модифицирующего фемтосекундного лазерного излучения, приводят к возникновению эффекта дихроизма в материале. Проводимость лазерно-модифицированных пленок определяется расположением анизотропных структур обоих типов относительно направления протекания тока. Однако величина их фотопроводимости не зависит от поляризации падающего света и не коррелирует с темновой проводимостью пленок. Анализ спектральных зависимостей фотопроводимости исследованных пленок показал, что на фотоэлектрические свойства пленок определяющее влияние оказывает распределение аморфной и кристаллической фаз материала.

Авторы выражают благодарность О.И. Конькову за представление образцов $a$-Si:H и А.А. Хомичу за измерение спектров КРС. Работа выполнена при финансовой поддержке Минобрнауки РФ (соглашение № 14.604.21.0085, идентификатор проекта RFMEFI60414X0085)

\section{Список литературы}

[1] Shah A.V., Schade Y.H., Vanecek M., Meier J., VallatSauvain E., Wyrsch N., Kroll U., Droz C., Bailat J. // Prog. Photovolt: Res. Appl. 2004. Vol. 12. P. 113-142.

[2] Yu N., Capasso F. // Nature Materials. 2014. Vol. 13. P. 139150.

[3] Drevinskas R., Beresna M., Gecevičius M., Khenkin M., Kazanskii A.G., Matulaitienè I., Niaura G., Konkov O.I., Terukov E.I., SvirkoYu.P., Kazansky P.G. // Appl. Phys. Lett. 2015. Vol. 106. N 17. P. 171106-1-171106-5.

[4] Shieh S., Chen Z., Dai B., Wang Y., Zaitsev A., Pan C. // Appl. Phys. Lett. 2004. Vol. 85. N 7. P. 1232-1234.
[5] Nayak B.K., Gupta M.C. // Appl. Phys. A. 2007. Vol. 89. N 3. P. 663-666.

[6] Timoshenko V.Yu., Osminkina L.A., Efimova A.I., Golovan L.A., Kashkarov P.K., Kovalev D., Künzner N., Gross E., Diener J., Koch F. // Phys. Rev. B. 2003. Vol. 67. N 11. P. 113405-1113405-4.

[7] Голубев В.Г., Давыдов В.Ю., Медведев А.В., Певцов А.Д., Феоктистов Н.А. // ФТТ. 1997. Т. 39. Вып. 8. С. 1348-1353.

[8] Khenkin M., Emelyanov A., Kazanskii A., Forsh P., Kon'kov O., Beresna M., Gecevicius M., Kazansky P. // Proc. SPIE. 2014. Vol. 9140. P. 914012-1-914012-7

[9] Emelyanov A.V., Khenkin M.V., Kazanskii A.G., Forsh P.A., Kashkarov P.K., Lyubin E.V., Khomich A.A., Gecevicius M., Beresnad M., Kazansky P.G. // Proc. SPIE. 2012. Vol. 8438. P. 84381-1-84381-8. 